\title{
Congruences for central factorial numbers modulo powers of prime
}

\author{
Haiqing Wang ${ }^{*}$ and Guodong Liu
}

*Correspondence:

Department of Mathematics,

Huizhou University,

Huizhou 516007,

Guangdong, People's

Republic of China

\begin{abstract}
Central factorial numbers are more closely related to the Stirling numbers than the other well-known special numbers, and they play a major role in a variety of branches of mathematics. In the present paper we prove some interesting congruences for central factorial numbers.
\end{abstract}

Keywords: Central factorial numbers of the first kind, Central factorial numbers of the second kind, Congruence, Stirling numbers

Mathematics Subject Classification: 11B68, 11B73, 05A10, 11B83

\section{Introduction and definitions}

Central factorial numbers are more closely related to the Stirling numbers than the other well-known special numbers, such as Bernoulli numbers, Euler numbers, trigonometric functions and their inverses. Properties of these numbers have been studied in different perspectives (see Butzer et al. 1989; Comtet 1974; Liu 2011; Merca 2012; Riordan 1968). Central factorial numbers play a major role in a variety of branches of mathematics (see Butzer et al. 1989; Chang and Ha 2009; Vogt 1989): to finite difference calculus, to approximation theory, to numerical analysis, to interpolation theory, in particular to Voronovskaja and Komleva-type expansions of trigonometric convolution integrals.

The central factorial numbers $t(n, k)(k \in \mathbb{Z})$ of the first kind and $T(n, k)(k \in \mathbb{Z})$ of the second kind are given by the following expansion formulas (see Butzer et al. 1989; Liu 2011; Riordan 1968)

$$
x^{[n]}=\sum_{k=0}^{n} t(n, k) x^{k}
$$

and

$$
x^{n}=\sum_{k=0}^{n} T(n, k) x^{[k]},
$$

respectively, where $\quad x^{[n]}=x\left(x+\frac{n}{2}-1\right)\left(x+\frac{n}{2}-2\right) \cdots\left(x+\frac{n}{2}-n+1\right), \quad n \in \mathbb{N}_{0}:=$ $\mathbb{N} \cup\{0\}, \mathbb{N}$ being the set of positive integers, $\mathbb{Z}$ being the set of integers.

(c) 2016 Wang and Liu. This article is distributed under the terms of the Creative Commons Attribution 4.0 International License (http://creativecommons.org/licenses/by/4.0/), which permits unrestricted use, distribution, and reproduction in any medium, provided you give appropriate credit to the original author(s) and the source, provide a link to the Creative Commons license, and indicate if changes were made. 
It follows from (1) that

$$
t(n, k)=t(n-2, k-2)-\frac{1}{4}(n-2)^{2} t(n-2, k)
$$

with

$$
\left(x^{2}-1^{2}\right)\left(x^{2}-2^{2}\right) \cdots\left(x^{2}-(n-1)^{2}\right)=\sum_{k=1}^{n} t(2 n, 2 k) x^{2 k-2} .
$$

Similarly, (2) gives

$$
T(n, k)=T(n-2, k-2)+\frac{1}{4} k^{2} T(n-2, k)
$$

with

$$
\frac{x^{2 k}}{\left(1-x^{2}\right)\left(1-(2 x)^{2}\right) \cdots\left(1-(k x)^{2}\right)}=\sum_{n=0}^{\infty} T(2 n, 2 k) x^{2 n}
$$

and

$$
k ! T(n, k)=\sum_{i=0}^{k}(-1)^{i}\left(\begin{array}{l}
k \\
i
\end{array}\right)\left(\frac{k}{2}-i\right)^{n} .
$$

Several papers obtain useful results on congruences of Stirling numbers, Bernoulli numbers and Euler numbers (see Chan and Manna 2010; Lengyel 2009; Sun 2005; Zhao et al. 2014). But only a few of congruences on central factorial numbers for odd prime moduli which can be found in (Riordan 1968, p. 236). For example, let $t_{n}(x)=\sum_{k=0}^{n} t(n, k) x^{k}$, then

$$
\begin{aligned}
& t_{p}(x) \equiv x^{p}-x \quad(\bmod p), \\
& t_{p+k}(x) \equiv t_{p}(x) \cdot t_{k}(x) \quad(\bmod p) .
\end{aligned}
$$

\section{Conclusions}

In the present paper we prove some other interesting congruences for central factorial numbers. In "Congruences for $T\left(a p^{m-1}(p-1)+r, k\right)$ modulo powers of prime $p$ " section, some congruence relations for $T\left(a p^{m-1}(p-1)+r, k\right)$ modulo powers of prime $p$ are established. For $a$ is odd, $m, k \in \mathbb{N}$ and $k \leq 2^{m-1} a$, we prove that

$$
k ! T\left(2^{m-1} a, k\right) \equiv \begin{cases}-2^{k-1} & \left(\bmod 2^{m}\right), \quad k \equiv 0 \quad(\bmod 4), \\ 2^{k-1} & \left(\bmod 2^{m}\right), \quad k \equiv 2 \quad(\bmod 4) .\end{cases}
$$

For $p$ is odd prime, $m, a, k \in \mathbb{N}, r \in \mathbb{N}_{0}, k \leq p-1$ and $r<p^{m-1}(p-1)$, in "Congruences for $T\left(a p^{m-1}(p-1)+r, k\right)$ modulo powers of prime $p$ " section we also show that

$$
T\left(a p^{m-1}(p-1)+r, k\right) \equiv T(r, k) \quad\left(\bmod p^{m}\right), \quad 1 \leq r<p^{m-1}(p-1)
$$


and

$$
k ! T\left(a p^{m-1}(p-1), k\right) \equiv(-1)^{\frac{k}{2}+1}\left(\begin{array}{l}
k \\
\frac{k}{2}
\end{array}\right) \quad\left(\bmod p^{m}\right), \quad \mathrm{k} \text { is even. }
$$

In "Congruences for $t\left(2 a p^{m}, 2 k\right)$ and $T\left(2 n, 2 a p^{m}\right)$ modulo powers of $p$ " section, congruences on $t\left(2 a p^{m}, 2 k\right)$ and $T\left(2 n, 2 a p^{m}\right)$ modulo powers of $p$ are derived. Moreover, the following results are obtained: (1) for $a, k, m \in \mathbb{N}, b \in \mathbb{N}_{0}$ and $2^{m-1} a \leq k \leq 2^{m} a$, we prove a congruence for $t\left(2^{m+1} a+2 b, 2 k\right)\left(\bmod 2^{m}\right)$; (2) for $a, n, m \in \mathbb{N}, b \in \mathbb{N}_{0}$ and $n \geq 2^{m} a$, we prove a congruence for $T\left(2 n, 2^{m+1} a+2 b\right)\left(\bmod 2^{m}\right)$; (3) for $p$ is a odd prime number and $a, k, m \in \mathbb{N}, b \in \mathbb{N}_{0}$, we deduce a congruence for $t\left(2 a p^{m}+2 b, 2 k\right)\left(\bmod p^{m}\right)$; (4) for $p$ is a odd prime number, $a, n, m \in \mathbb{N}, b \in \mathbb{N}_{0}$, we deduce a congruence for $T\left(2 n, 2 a p^{m}+2 b\right)\left(\bmod p^{m}\right)$.

\section{Congruences for $T\left(a p^{m-1}(p-1)+r, k\right)$ modulo powers of prime $p$}

Theorem 1 For $a$ is odd, $m, k \in \mathbb{N}$ and $k \leq 2^{m-1} a$, we have

$$
k ! T\left(2^{m-1} a, k\right) \equiv\left\{\begin{array}{l}
-2^{k-1} \quad\left(\bmod 2^{m}\right), \quad k \equiv 0 \quad(\bmod 4) \\
2^{k-1} \quad\left(\bmod 2^{m}\right), \quad k \equiv 2 \quad(\bmod 4)
\end{array}\right.
$$

Proof Using Euler's Theorem, $\varphi\left(2^{m}\right)=2^{m-1}$. Therefore, by Fermat's Little Theorem, we get $c^{\varphi\left(2^{m}\right)}=c^{2^{m-1}} \equiv 1\left(\bmod 2^{m}\right)$ if $c$ is odd. Observe that, when $c$ is even, $c^{2^{m-1}} \equiv 0\left(\bmod 2^{m}\right)$.

Then by $(7)$, if $k \equiv 0(\bmod 4)$, we yield

$$
\begin{aligned}
k ! T\left(2^{m-1} a, k\right) & =\sum_{i=0}^{k}(-1)^{i}\left(\begin{array}{c}
k \\
i
\end{array}\right)\left(\frac{k}{2}-i\right)^{2^{m-1} a} \\
& \equiv \sum_{i=1, i \text { odd }}^{k}(-1)^{i}\left(\begin{array}{l}
k \\
i
\end{array}\right) \\
& =-2^{k-1}\left(\bmod 2^{m}\right) .
\end{aligned}
$$

If $k \equiv 2(\bmod 4)$, we have

$$
\begin{aligned}
k ! T\left(2^{m-1} a, k\right) & =\sum_{i=0}^{k}(-1)^{i}\left(\begin{array}{l}
k \\
i
\end{array}\right)\left(\frac{k}{2}-i\right)^{2^{m-1} a} \\
& \equiv \sum_{i=0, i \text { even }}^{k}(-1)^{i}\left(\begin{array}{l}
k \\
i
\end{array}\right) \\
& =2^{k-1}\left(\bmod 2^{m}\right) .
\end{aligned}
$$

This completes the proof of Theorem 1 . 
Remark By Theorem 1 and (5), we readily get

$$
k ! T\left(2^{m-1} a+2, k\right) \equiv\left\{\begin{array}{l}
-k \cdot 2^{k-3} \quad\left(\bmod 2^{m}\right), \quad k \equiv 0 \quad(\bmod 4) \\
k \cdot 2^{k-3} \quad\left(\bmod 2^{m}\right), \quad k \equiv 2 \quad(\bmod 4)
\end{array}\right.
$$

Theorem 2 For $p$ is odd prime, $m, a, k \in \mathbb{N}, r \in \mathbb{N}_{0}, k \leq p-1$ and $r<p^{m-1}(p-1)$, we have

$$
\begin{aligned}
& T\left(a p^{m-1}(p-1)+r, k\right) \equiv T(r, k) \quad\left(\bmod p^{m}\right), \quad 1 \leq r<p^{m-1}(p-1), \\
& k ! T\left(a p^{m-1}(p-1), k\right) \equiv(-1)^{\frac{k}{2}+1}\left(\begin{array}{c}
k \\
\frac{k}{2}
\end{array}\right) \quad\left(\bmod p^{m}\right), \quad \mathrm{k} \text { is even. }
\end{aligned}
$$

Proof By Euler's Theorem and Fermat's Little Theorem, we get $a^{\varphi\left(p^{m}\right)}=$ $a^{p^{m-1}(p-1)} \equiv 1\left(\bmod p^{m}\right)$ if $(a, p)=1$, where $(a, p)$ is the greatest common factor of $a$ and $p$. Then by $(7)$ and noting that $(k-2 i, p)=1$, we get

$$
\begin{aligned}
k ! T\left(a p^{m-1}(p-1)+r, k\right) & =\sum_{i=0}^{k}(-1)^{i}\left(\begin{array}{l}
k \\
i
\end{array}\right)\left(\frac{k}{2}-i\right)^{a p^{m-1}(p-1)+r} \\
& \equiv \sum_{i=0}^{k}(-1)^{i}\left(\begin{array}{c}
k \\
i
\end{array}\right)\left(\frac{k}{2}-i\right)^{r} \\
& =k ! T(r, k)\left(\bmod p^{m}\right) .
\end{aligned}
$$

Observe that $(k !, p)=1$. Hence,

$$
T\left(a p^{m-1}(p-1)+r, k\right) \equiv T(r, k) \quad\left(\bmod p^{m}\right)
$$

The proof of (12) is complete. If $r=0$, then $k$ is even. Therefore,

$$
\begin{aligned}
k ! T\left(a p^{m-1}(p-1), k\right) & =\sum_{i=0}^{k}(-1)^{i}\left(\begin{array}{l}
k \\
i
\end{array}\right)\left(\frac{k}{2}-i\right)^{a p^{m-1}(p-1)} \\
& \equiv \sum_{i=0}^{k}(-1)^{i}\left(\begin{array}{l}
k \\
i
\end{array}\right)-(-1)^{\frac{k}{2}}\left(\begin{array}{c}
k \\
\frac{k}{2}
\end{array}\right) \\
& =(-1)^{\frac{k}{2}+1}\left(\begin{array}{l}
k \\
\frac{k}{2}
\end{array}\right) \quad\left(\bmod p^{m}\right) .
\end{aligned}
$$

The proof of (13) is complete. This completes the proof of Theorem 2.

As a direct consequence of Theorem 2, we have the following corollary.

Corollary 3 For $p$ is odd prime, $a, k \in \mathbb{N}$ and $r \in \mathbb{N}_{0}$, we have

$$
T(a(p-1)+r, p) \equiv\left\{\begin{array}{lll}
0 & (\bmod p), & 3 \leq r \leq p-2 \\
1 & (\bmod p), & r=1
\end{array}\right.
$$




$$
\begin{aligned}
& T(a(p-1)+r, p-1) \equiv\left\{\begin{array}{lll}
0 & (\bmod p), & 1 \leq r \leq p-1, \\
1 & (\bmod p), & r=0 .
\end{array}\right. \\
& T(p+2, k+2) \equiv T(p, k) \equiv 0 \quad(\bmod p), \quad 3 \leq k \leq p-1 . \\
& T(2 p+2, k+2) \equiv T(2 p, k) \equiv 0 \quad(\bmod p), \quad 4 \leq k \leq p-1 .
\end{aligned}
$$

Proof By setting $m=1$ in (12) and using (5), we have

$$
\begin{aligned}
T(a(p-1)+r, p) & \equiv T(a(p-1)+r-2, p-2) \\
& \equiv T(r-2, p-2)=0 \quad(\bmod p), \quad(3 \leq r \leq p-2), \\
T(a(p-1)+1, p) & \equiv T(a(p-1)-1, p-2) \\
& \equiv T(p-2, p-2)=1 \quad(\bmod p) .
\end{aligned}
$$

The proof of (14) is complete. Setting $m=1$ and $k=p-1$ in (12), we can readily get

$$
T(a(p-1)+r, p-1) \equiv 0 \quad(\bmod p) .
$$

Setting $m=1$ and $k=p-1$ in (13), and noting that $(-1)^{j}\left(\begin{array}{c}p-1 \\ j\end{array}\right) \equiv 1$ $(\bmod p) \quad(j=0,1,2, \ldots, p-1),(p-1) ! \equiv-1(\bmod p)$, we have

$$
T(a(p-1), p-1) \equiv 1 \quad(\bmod p) .
$$

The proof of (15) is complete. If $m=1$ and $a=r$ in (12), then

$$
T(r p, k) \equiv T(r, k) \quad(\bmod p)
$$

Taking $r=1,2$ in (18) and using (5), we immediately get (16) and (17). This completes the proof of Corollary 3.

\section{Congruences for $t\left(2 a p^{m}, 2 k\right)$ and $T\left(2 n, 2 a p^{m}\right)$ modulo powers of $p$}

To establish the main results in this section, we need to introduce the following lemmas.

Lemma 4 If $m \in \mathbb{N}$, then

$$
\begin{aligned}
& \prod_{i=1}^{2^{m-1}}\left(1-((2 i-1) x)^{2}\right) \equiv\left(1-x^{2}\right)^{2^{m-1}} \quad\left(\bmod 2^{m}\right) \\
& \prod_{i=1}^{2^{m-1}}\left(1-(2 i x)^{2}\right) \equiv 1 \quad\left(\bmod 2^{m}\right)
\end{aligned}
$$

Proof We prove this lemma by induction on $m$. We see that (19) is true for $m=1$. Assume that it is true for $m=1,2, \ldots, j-1$. Then 


$$
\begin{aligned}
& \prod_{i=1}^{2^{j}}\left(1-((2 i-1) x)^{2}\right) \\
& \quad=\prod_{i=1}^{2^{j-1}}\left(1-((2 i-1) x)^{2}\right)\left(1-\left(\left(2^{j}+2 i-1\right) x\right)^{2}\right) \\
& \quad=\prod_{i=1}^{2^{j-1}}\left[\left(1-((2 i-1) x)^{2}\right)^{2}-2^{j+1} x^{2}\left(2^{j-1}+2 i-1\right)\left(1-((2 i-1) x)^{2}\right)\right] \\
& \quad \equiv\left(\prod_{i=1}^{2^{j-1}}\left(1-((2 i-1) x)^{2}\right)\right)^{2}\left(\bmod 2^{j+1}\right) .
\end{aligned}
$$

For any polynomials $A(x), \quad B(x)$, we have $A(x) \equiv B(x)\left(\bmod 2^{m}\right) \rightarrow(A(x))^{2}$ $\equiv(B(x))^{2}\left(\bmod 2^{m+1}\right)$, so we obtain the desired result. That is,

$$
\prod_{i=1}^{2^{j}}\left(1-((2 i-1) x)^{2}\right) \equiv\left(\prod_{i=1}^{2^{j-1}}\left(1-((2 i-1) x)^{2}\right)\right)^{2} \equiv\left(1-x^{2}\right)^{2^{j}} \quad\left(\bmod 2^{j+1}\right)
$$

The proof of (19) is complete. Similarly, we can prove (20) as follows.

$$
\begin{aligned}
\prod_{i=1}^{2^{j}}\left(1-(2 i x)^{2}\right) & =\prod_{i=1}^{2^{j-1}}\left(1-(2 i x)^{2}\right)\left(1-\left(\left(2^{j}+2 i\right) x\right)^{2}\right) \\
& =\prod_{i=1}^{2^{j-1}}\left[\left(1-(2 i x)^{2}\right)^{2}-2^{j+1} x^{2}\left(2^{j-1}+2 i\right)\left(1-(2 i x)^{2}\right)\right] \\
& \equiv\left(\prod_{i=1}^{2^{j-1}}\left(1-(2 i x)^{2}\right)\right)^{2}\left(\bmod 2^{j+1}\right) \\
& \equiv 1 \quad\left(\bmod 2^{j+1}\right) .
\end{aligned}
$$

This completes the proof of Lemma 4.

Similarly, we can get the following results.

Lemma 5 If $m \in \mathbb{N}$, then

$$
\begin{aligned}
& \prod_{i=1}^{2^{m-1}}\left(x^{2}-(2 i-1)^{2}\right) \equiv\left(x^{2}-1\right)^{2^{m-1}} \quad\left(\bmod 2^{m}\right), \\
& \prod_{i=1}^{2^{m-1}}\left(x^{2}-(2 i)^{2}\right) \equiv x^{2 m} \quad\left(\bmod 2^{m}\right) .
\end{aligned}
$$

We are now ready to state the following theorems. 
Theorem 6 Let $a, b, k, m \in \mathbb{N}$ and $2^{m-1} a \leq k \leq 2^{m} a$ then

$$
\begin{aligned}
& t\left(2^{m+1} a, 2 k\right) \equiv(-1)^{k-2^{m-1} a}\left(\begin{array}{c}
2^{m-1} a \\
k-2^{m-1} a
\end{array}\right) \quad\left(\bmod 2^{m}\right), \\
& t\left(2^{m+1} a+2 b, 2 k\right) \equiv \sum_{j=1}^{k} t\left(2^{m+1} a, 2 j\right) t(2 b, 2 k-2 j) \quad\left(\bmod 2^{m}\right) .
\end{aligned}
$$

Proof By (4) and Lemma 5, we find that

$\sum_{k=1}^{2^{m} a} t\left(2^{m+1} a, 2 k\right) x^{2 k-2}\left(x^{2}-\left(2^{m} a\right)^{2}\right)=\left(x^{2}-1^{2}\right) \cdots\left(x^{2}-\left(2^{m} a-1\right)^{2}\right)\left(x^{2}-\left(2^{m} a\right)^{2}\right)$.

Thus

$$
\begin{aligned}
\sum_{k=1}^{2^{m} a} t\left(2^{m+1} a, 2 k\right) x^{2 k} & \equiv\left(\prod_{i=1}^{2^{m}}\left(x^{2}-i^{2}\right)\right)^{a} \\
& =\left(\prod_{i=1}^{2^{m-1}}\left(x^{2}-(2 i-1)^{2}\right) \prod_{i=1}^{2^{m-1}}\left(x^{2}-(2 i)^{2}\right)\right)^{a} \\
& \equiv\left(x^{2}-1\right)^{2^{m-1} a} x^{2^{m} a} \\
& =\sum_{k=0}^{2^{m-1} a}(-1)^{k}\left(\begin{array}{c}
2^{m-1} a \\
k
\end{array}\right) x^{2^{m} a+2 k} \\
& =\sum_{k=2^{m-1} a}^{2^{m} a}(-1)^{k}\left(\begin{array}{c}
2^{m-1} a \\
k-2^{m-1} a
\end{array}\right) x^{2 k} \quad\left(\bmod 2^{m}\right)
\end{aligned}
$$

This completes the proof of (23). For (24), we can prove this as follows.

$$
\begin{aligned}
& \sum_{k=1}^{2^{m} a+b} t\left(2^{m+1} a+2 b, 2 k\right) x^{2 k-2} \\
& =\left(x^{2}-1^{2}\right) \cdots\left(x^{2}-\left(2^{m} a\right)^{2}\right)\left(x^{2}-\left(2^{m} a+1\right)^{2}\right) \cdots\left(x^{2}-\left(2^{m} a+b-1\right)^{2}\right) \\
& \equiv\left(x^{2}-1^{2}\right) \cdots\left(x^{2}-\left(2^{m} a\right)^{2}\right)\left(x^{2}-1\right)^{2} \cdots\left(x^{2}-(b-1)^{2}\right) \\
& \equiv \sum_{k=1}^{2^{m} a} t\left(2^{m+1} a, 2 k\right) x^{2 k} \sum_{k=1}^{b} t(2 b, 2 k) x^{2 k-2} \\
& =\sum_{k=2}^{2^{m} a+b} \sum_{j=1}^{k} t\left(2^{m+1} a, 2 j\right) t(2 b, 2 k-2 j) x^{2 k-2} \quad\left(\bmod 2^{m}\right) .
\end{aligned}
$$

This completes the proof of Theorem 6. 
Remark Taking $a=1$ and $k=2^{m-1}, 2^{m-1}+1,2^{m-1}+2$ in (23), we readily get

$$
\begin{aligned}
t\left(2^{m+1}, 2^{m}\right) & \equiv 1 \quad\left(\bmod 2^{m}\right), \\
t\left(2^{m+1}, 2^{m}+2\right) & \equiv 2^{m-1} \quad\left(\bmod 2^{m}\right), \\
t\left(2^{m+1}, 2^{m}+4\right) & \equiv 3 \cdot 2^{m-2} \quad\left(\bmod 2^{m}\right), \quad m \geq 3 .
\end{aligned}
$$

Theorem 7 Let $a, b, n, m \in \mathbb{N}$ and $n \geq 2^{m} a$, then

$$
\begin{aligned}
& T\left(2 n, 2^{m+1} a\right) \equiv\left(\begin{array}{c}
n-2^{m-1} a-1 \\
n-2^{m} a
\end{array}\right) \quad\left(\bmod 2^{m}\right) \\
& T\left(2 n, 2^{m+1} a+2 b\right) \equiv \sum_{j=0}^{n} T\left(2 j, 2^{m+1} a\right) T(2 n-2 j, 2 b) \quad\left(\bmod 2^{m}\right) .
\end{aligned}
$$

Proof By (6) and Lemma 4, we have

$$
\begin{aligned}
\sum_{n=0}^{\infty} T\left(2 n, 2^{m+1} a\right) x^{2 n} & =\prod_{i=1}^{2^{m} a} \frac{x^{2}}{\left(1-(i x)^{2}\right)} \\
& \equiv\left(\prod_{i=1}^{2^{m}} \frac{x^{2}}{\left(1-(i x)^{2}\right)}\right)^{a} \\
& \equiv x^{2^{m+1} a}\left(\frac{1}{\prod_{i=1}^{2^{m-1}}\left(1-((2 i-1) x)^{2}\right) \prod_{i=1}^{2^{m-1}}\left(1-(2 i)^{2}\right)}\right)^{a} \\
& \equiv x^{2^{m+1} a} \frac{1}{\left(1-x^{2}\right)^{2^{m-1} a}} \\
& =\sum_{n=0}^{\infty}\left(\begin{array}{c}
n+2^{m-1} a-1 \\
n
\end{array}\right) x^{2^{m+1} a+2 n} \\
& =\sum_{n=2^{m} a}^{\infty}\left(\begin{array}{c}
n-2^{m-1} a-1 \\
n-2^{m} a
\end{array}\right) x^{2 n} \quad\left(\bmod 2^{m}\right) .
\end{aligned}
$$

This completes the proof of (25). For (26), we can prove this as follows.

$$
\begin{aligned}
\sum_{n=0}^{\infty} T\left(2 n, 2^{m+1} a+2 b\right) x^{2 n} & =\prod_{i=1}^{2^{m} a+b} \frac{x^{2}}{\left(1-(i x)^{2}\right)} \\
& \equiv \prod_{i=1}^{2^{m} a} \frac{x^{2}}{\left(1-(i x)^{2}\right)} \prod_{i=1}^{b} \frac{x^{2}}{\left(1-(i x)^{2}\right)} \\
& =\sum_{n=0}^{\infty} T\left(2 n, 2^{m+1} a\right) x^{2 n} \sum_{n=0}^{\infty} T(2 n, 2 b) x^{2 n} \\
& =\sum_{n=0}^{\infty} \sum_{j=0}^{n} T\left(2 j, 2^{m+1} a\right) T(2 n-2 j, 2 b) x^{2 n} \quad\left(\bmod 2^{m}\right)
\end{aligned}
$$


This completes the proof of Theorem 7.

Remark Taking $a=1$ and $n=2^{m}+1,2^{m}+2$ in (25), we readily get

$$
\begin{aligned}
& T\left(2^{m+1}+2,2^{m+1}\right) \equiv 2^{m-1} \quad\left(\bmod 2^{m}\right), \\
& T\left(2^{m+1}+4,2^{m+1}\right) \equiv 2^{m-2} \quad\left(\bmod 2^{m}\right), \quad m \geq 3 .
\end{aligned}
$$

Lemma 8 If $p$ is a odd prime number and $m \in \mathbb{N}$, then

$$
\prod_{i=1}^{p^{m}}\left(1-(i x)^{2}\right) \equiv\left(1-x^{p-1}\right)^{2 p^{m-1}} \quad\left(\bmod p^{m}\right) .
$$

Proof Apparently, by Lagrange congruence, we have

$$
(1-x)(1-2 x) \cdots(1-(p-1) x)(1-p x) \equiv\left(1-x^{p-1}\right) \quad(\bmod p)
$$

and

$$
(1+x)(1+2 x) \cdots(1+(p-1) x)(1+p x) \equiv\left(1-x^{p-1}\right) \quad(\bmod p) .
$$

Thus

$$
\left(1-x^{2}\right)\left(1-(2 x)^{2}\right) \cdots\left(1-(p x)^{2}\right) \equiv\left(1-x^{p-1}\right)^{2} \quad(\bmod p) .
$$

Hence (27) is true for the case $m=1$.

Suppose that (27) is true for some $m \geq 1$. Then for the case $m+1$,

$$
\begin{aligned}
& \prod_{i=1}^{p^{m+1}}\left(1-(i x)^{2}\right) \\
& =\prod_{i=1}^{p^{m}}\left(1-(i x)^{2}\right)\left(1-\left(p^{m}+i x\right)^{2}\right)\left(1-\left(2 p^{m}+i x\right)^{2}\right) \cdots\left(1-\left((p-1) p^{m}+i x\right)^{2}\right) \\
& =\prod_{i=1}^{p^{m}}\left[\left(1-(i x)^{2}\right)^{p}-\left(1-(i x)^{2}\right)^{p-1}\left(\sum_{j=1}^{p-1}\left(j p^{m}\right)^{2}+2 j p^{m} i x\right)\right. \\
& \left.\quad+\text { terms involving powers of } p^{2 m} \text { and higher }\right] .
\end{aligned}
$$

For any prime $p$ and polynomials $A(x), B(x)$, we have $A(x) \equiv B(x)\left(\bmod p^{m}\right)$. This implies that $(A(x))^{p} \equiv(B(x))^{p}\left(\bmod p^{m+1}\right)$. With $\sum_{j=1}^{p-1}\left(j p^{m}\right)^{2}+2 j p^{m} i x \equiv 0\left(\bmod p^{m+1}\right)$, we obtain the desired result. That is,

$$
\prod_{i=1}^{p^{m+1}}\left(1-(i x)^{2}\right) \equiv\left(\prod_{i=1}^{p^{m}} 1-(i x)^{2}\right)^{p} \equiv\left(1-x^{p-1}\right)^{2 p^{m}} \quad\left(\bmod p^{m+1}\right) .
$$

This completes the proof of Lemma 8. 
Similarly, we get the following results.

Lemma 9 If $p$ is a odd prime number and $m \in \mathbb{N}$, then

$$
\prod_{i=1}^{p^{m}}\left(x^{2}-i^{2}\right) \equiv\left(x^{p}-x\right)^{2 p^{m-1}} \quad\left(\bmod p^{m}\right)
$$

Theorem 10 Let $p$ is a odd prime number and $a, b, k, m \in \mathbb{N}$, then

$$
\begin{aligned}
& t\left(2 a p^{m}, 2 k\right) \equiv(-1)^{\frac{2 k-2 a p^{m-1}}{p-1}}\left(\begin{array}{c}
2 a p^{m-1} \\
\frac{2 k-2 a p^{m-1}}{p-1}
\end{array}\right) \quad\left(\bmod p^{m}\right), \\
& t\left(2 a p^{m}+2 b, 2 k\right) \equiv \sum_{j=1}^{k} t\left(2 a p^{m}, 2 j\right) t(2 b, 2 k-2 j) \quad\left(\bmod p^{m}\right),
\end{aligned}
$$

where $2 k \equiv 2 a p^{m-1}(\bmod p-1)$.

Proof By (4) and Lemma 9, we find that

$$
\sum_{k=1}^{a p^{m}} t\left(2 a p^{m}, 2 k\right) x^{2 k-2}\left(x^{2}-\left(a p^{m}\right)^{2}\right)=\left(x^{2}-1^{2}\right) \cdots\left(x^{2}-\left(a p^{m}-1\right)^{2}\right)\left(x^{2}-\left(a p^{m}\right)^{2}\right) .
$$

Thus

$$
\begin{aligned}
\sum_{k=1}^{a p^{m}} t\left(2 a p^{m}, 2 k\right) x^{2 k} & \equiv\left(\prod_{i=1}^{p^{m}}\left(x^{2}-i^{2}\right)\right)^{a} \\
& \equiv\left(x^{p}-x\right)^{2 a p^{m-1}} \\
& =\sum_{k=0}^{2 a p^{m-1}}(-1)^{k}\left(\begin{array}{c}
2 a p^{m-1} \\
k
\end{array}\right) x^{2 a p^{m-1}+(p-1) k} \\
& =\sum_{k=a p^{m-1}}^{a p^{m}}(-1)^{\frac{2 k-2 a p^{m-1}}{p-1}}\left(\begin{array}{c}
2 a p^{m-1} \\
\frac{2 k-2 a p^{m-1}}{p-1}
\end{array}\right) x^{2 k} \quad\left(\bmod p^{m}\right),
\end{aligned}
$$

where $2 k \equiv 2 a p^{m-1}(\bmod p-1)$. This completes the proof of $(29)$.

By (4) we get

$$
\begin{aligned}
& \sum_{k=1}^{a p^{m}+b} t\left(2 a p^{m}+2 b, 2 k\right) x^{2 k-2} \\
& =\left(x^{2}-1^{2}\right) \cdots\left(x^{2}-\left(a p^{m}\right)^{2}\right)\left(x^{2}-\left(a p^{m}+1\right)^{2}\right) \cdots\left(x^{2}-\left(a p^{m}+b-1\right)^{2}\right) \\
& \left.\equiv\left(x^{2}-1^{2}\right) \cdots\left(x^{2}-\left(a p^{m}\right)^{2}\right)\left(x^{2}-1\right)^{2}\right) \cdots\left(x^{2}-(b-1)^{2}\right) \\
& =\sum_{k=1}^{a p^{m}} t\left(2 a p^{m}, 2 k\right) x^{2 k} \sum_{k=1}^{b} t(2 b, 2 k) x^{2 k-2} \\
& =\sum_{k=2}^{a p^{m}+b} \sum_{j=1}^{k} t\left(2 a p^{m}, 2 j\right) t(2 b, 2 k-2 j) x^{2 k-2} \quad\left(\bmod p^{m}\right) .
\end{aligned}
$$


This completes the proof of Theorem 10 .

Remark Taking $a=1$ and $2 k=2 p^{m-1}, 2 p^{m-1}+(p-1), 2 p^{m-1}+2(p-1)$ in (29), we readily get

$$
\begin{aligned}
& t\left(2 p^{m}, 2 p^{m-1}\right) \equiv 1 \quad\left(\bmod p^{m}\right), \\
& t\left(2 p^{m}, 2 p^{m-1}+(p-1)\right) \equiv-2 p^{m-1} \quad\left(\bmod p^{m}\right), \\
& t\left(2 p^{m}, 2 p^{m-1}+2(p-1)\right) \equiv 2 p^{2 m-2}-p^{m-1} \quad\left(\bmod p^{m}\right) .
\end{aligned}
$$

Obviously ,

$$
\begin{aligned}
& t(2 p, 2) \equiv 1 \quad(\bmod p) \\
& t(2 p, p+1) \equiv-2 \quad(\bmod p) .
\end{aligned}
$$

If $u, v \in \mathbb{N}, 1 \leq u<2 p^{v}$. By setting $m=1, a=p^{v}, 2 k=2 p^{v}+u(p-1)$ in (29), and noting that $\left(\begin{array}{c}p \\ j\end{array}\right) \equiv 1(\bmod p) \quad(j=0,1,2, \ldots, p-1)$ with $\left(\begin{array}{c}i p+r \\ j p+s\end{array}\right) \equiv\left(\begin{array}{l}i \\ j\end{array}\right)\left(\begin{array}{l}r \\ s\end{array}\right)(\bmod p) \quad(i \geq j)$, we have

$$
t\left(2 p^{v+1}, 2 p^{v}+u(p-1)\right) \equiv(-1)^{u}\left(\begin{array}{c}
2 p^{v} \\
u
\end{array}\right) \equiv 0 \quad(\bmod p)
$$

The following corollary is a direct consequence of Theorem 10.

Corollary 11 Let $p$ be a odd prime and $\alpha$ be a positive integer. Then for any $1 \leq k \leq p^{\alpha}(p-1)$, we have

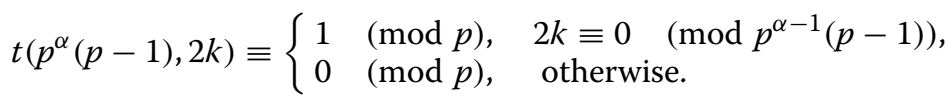

Proof Let $m=1,2 a=p^{\alpha-1}(p-1)$ in (29) of Theorem 10. Then we have

$$
\sum_{k=1}^{\frac{p^{\alpha}(p-1)}{2}} t\left(p^{\alpha}(p-1), 2 k\right) x^{2 k} \equiv \sum_{j=0}^{p^{\alpha-1}(p-1)}(-1)^{j}\left(\begin{array}{c}
p^{\alpha-1}(p-1) \\
j
\end{array}\right) x^{p^{\alpha-1}(p-1)+(p-1) j} \quad(\bmod p) .
$$

By the Lucas congruence, we obtain

$$
\left(\begin{array}{c}
p^{\alpha-1}(p-1) \\
j
\end{array}\right) \equiv\left\{\begin{array}{l}
\left(\begin{array}{c}
p-1 \\
\frac{j}{p^{\alpha-1}}
\end{array}\right) \quad(\bmod p), \quad j \equiv 0 \quad\left(\bmod p^{\alpha-1}\right) \\
0 \quad(\bmod p), \quad \text { otherwise }
\end{array}\right.
$$

With

$$
(-1)^{j}\left(\begin{array}{c}
p-1 \\
j
\end{array}\right) \equiv 1 \quad(\bmod p) \quad(j=0,1,2, \ldots, p-1),
$$


we can deduce

$$
\begin{aligned}
\sum_{k=1}^{\frac{p^{\alpha}(p-1)}{2}} t\left(p^{\alpha}(p-1), 2 k\right) x^{2 k} & \equiv \sum_{j=0}^{p-1}(-1)^{j}\left(\begin{array}{c}
p^{\alpha-1}(p-1) \\
p^{\alpha-1} j
\end{array}\right) x^{p^{\alpha-1}(p-1)(j+1)} \\
& \equiv \sum_{j=1}^{p}(-1)^{j-1}\left(\begin{array}{c}
p-1 \\
j-1
\end{array}\right) x^{p^{\alpha-1}(p-1) j} \\
& \equiv \sum_{j=1}^{p} x^{p^{\alpha-1}(p-1) j \quad(\bmod p)}
\end{aligned}
$$

which is obviously equivalent to (37).

The following theorem includes the congruence relations for $T\left(2 n, 2 a p^{m}\right)$ and $T\left(2 n, 2 a p^{m}+2 b\right.$.

Theorem 12 If $p$ is a odd prime number, $a, b, n, m \in \mathbb{N}$, then

$$
T\left(2 n, 2 a p^{m}\right) \equiv\left(\begin{array}{c}
\frac{2 n-2 a p^{m-1}}{p-1}-1 \\
\frac{2 n-2 a p^{m}}{p-1}
\end{array}\right) \quad\left(\bmod p^{m}\right)
$$

and

$$
T\left(2 n, 2 a p^{m}+2 b\right) \equiv \sum_{j=0}^{n} T\left(2 j, 2 a p^{m}\right) T(2 n-2 j, 2 b) \quad\left(\bmod p^{m}\right)
$$

where $2 n \equiv 2 a p^{m}(\bmod p-1)$

Proof By (6) and Lemma 8, we have

$$
\begin{aligned}
\sum_{n=0}^{\infty} T\left(2 n, 2 a p^{m}\right) x^{2 n} & =\prod_{i=1}^{a p^{m}} \frac{x^{2}}{\left(1-(i x)^{2}\right)} \\
& \equiv\left(\prod_{i=1}^{p^{m}} \frac{x^{2}}{\left(1-(i x)^{2}\right)}\right)^{a} \\
& \equiv x^{2 a p^{m}} \frac{1}{\left(1-x^{p-1}\right)^{2 a p^{m-1}}} \\
& =\sum_{n=0}^{\infty}\left(\begin{array}{c}
n+2 a p^{m-1}-1 \\
n
\end{array}\right) x^{2 a p^{m}+(p-1) n} \\
& =\sum_{n=a p^{m}}^{\infty}\left(\begin{array}{c}
\frac{2 n-2 a p^{m-1}}{p-1}-1 \\
\frac{2 n-2 a p^{m}}{p-1}
\end{array}\right) x^{2 n} \quad\left(\bmod p^{m}\right)
\end{aligned}
$$

This completes the proof of (38). For (39), we can prove this as follows. 


$$
\begin{aligned}
\sum_{n=0}^{\infty} T\left(2 n, 2 a p^{m}+2 b\right) x^{2 n} & =\prod_{i=1}^{a p^{m}+b} \frac{x^{2}}{\left(1-(i x)^{2}\right)} \\
& \equiv \prod_{i=1}^{a p^{m}} \frac{x^{2}}{\left(1-(i x)^{2}\right)} \prod_{i=1}^{b} \frac{x^{2}}{\left(1-(i x)^{2}\right)} \\
& =\sum_{n=0}^{\infty} T\left(2 n, 2 a p^{m}\right) x^{2 n} \sum_{n=0}^{\infty} T(2 n, 2 b) x^{2 n} \\
& =\sum_{n=0}^{\infty} \sum_{j=0}^{n} T\left(2 j, 2 a p^{m}\right) T(2 n-2 j, 2 m) x^{2 n} \quad\left(\bmod p^{m}\right) .
\end{aligned}
$$

This completes the proof of Theorem 12.

Remark Taking $a=1$ and $2 n=2 p^{m}+(p-1), 2 p^{m}+2(p-1)$ in (38), we have

$$
\begin{aligned}
& T\left(2 p^{m}+(p-1), 2 p^{m}\right) \equiv 2 p^{m-1} \quad\left(\bmod p^{m}\right) \\
& T\left(2 p^{m}+2(p-1), 2 p^{m}\right) \equiv 2 p^{2 m-2}+p^{m-1} \quad\left(\bmod p^{m}\right) .
\end{aligned}
$$

Obviously ,

$$
\begin{aligned}
& T(3 p-1,2 p) \equiv 2 \quad(\bmod p) \\
& T(4 p-2,2 p) \equiv 3 \quad(\bmod p) .
\end{aligned}
$$

If $u \in \mathbb{N}_{0}, v \in \mathbb{N}$. By setting $m=1, a=p^{v-1}, 2 n=2 p^{u+v}$ in (38), and noting that $\left(\begin{array}{l}i p+r \\ j p+s\end{array}\right) \equiv\left(\begin{array}{l}i \\ j\end{array}\right)\left(\begin{array}{l}r \\ s\end{array}\right)(\bmod p) \quad(i \geq j)$, we have

$$
\begin{aligned}
T\left(2 p^{u+v}, 2 p^{v}\right) & \equiv\left(\begin{array}{c}
2 p^{v-1} \sum_{i=0}^{u} p^{i}-1 \\
2 p^{v-1}-1
\end{array}\right) \\
& =\frac{1}{\sum_{i=0}^{u} p^{i}}\left(\begin{array}{c}
2 p^{v-1} \sum_{i=0}^{u} p^{i} \\
2 p^{v-1}
\end{array}\right) \\
& \equiv \frac{1}{\sum_{i=0}^{u} p^{i}}\left(\begin{array}{c}
2 \sum_{i=0}^{u} p^{i} \\
2
\end{array}\right) \\
& \equiv 1 \quad(\bmod p) .
\end{aligned}
$$

That is,

$$
T\left(2 p^{u+v}, 2 p^{v}\right) \equiv 1 \quad(\bmod p) .
$$

Authors' contributions

Both authors read and approved the final manuscript.

\section{Competing interests}

The authors declare that they have no competing interests.

Received: 8 October 2015 Accepted: 18 March 2016

Published online: 31 March 2016 
References

Butzer PL, Schmidt M, Stark EL, Vogt L (1989) Central factorial numbers; their main properties and some applications. Numer Funct Anal Optim 10:419-488

Chang CH, Ha CW (2009) Central factorial numbers and values of Bernoulli and Euler polynomials at rationals. Numer Funct Anal Optim 30(3-4):214-226

Comtet L (1974) Advanced combinatorics: the art of finite and infinite expansions (translated from the French by J. W. Nienhuys). Reidel, Dordrecht and Boston

Chan O-Y, Manna DV (2010) Congruences for Stirling numbers of the second kind. Contemp Math 517:97-111

Liu GD (2011) The D numbers and the central factorial numbers. Publ Math Debr 79(1-2):41-53

Lengyel T (2009) On the 2-adic order of Stirling numbers of the second kind and their differences. In: DMTCS Proc AK, pp $561-572$

Merca Mircea (2012) A special case of the generalized Girard-Waring formula. J Integer Seq, Article 12.5.7, 15:7

Riordan J (1968) Combinatorial identities. Wiley, New York

Sun ZW (2005) On Euler numbers modulo powers of two. J Number Theory 115:371-380

Vogt L (1989) Approximation by linear combinations of positive convolution integrals. J Approx Theory 57(2):178-201

Zhao J, Hong S, Zhao W (2014) Divisibility by 2 of Stirling numbers of the second kind and their differences. J Number Theory 140:324-348

\section{Submit your manuscript to a SpringerOpen ${ }^{\circ}$ journal and benefit from:}

- Convenient online submission

- Rigorous peer review

- Immediate publication on acceptance

- Open access: articles freely available online

- High visibility within the field

- Retaining the copyright to your article

Submit your next manuscript at $>$ springeropen.com 